\title{
Non-Fermi liquid and pairing in electron-doped cuprates
}

\author{
P. Krotkov ${ }^{1}$ and Andrey V. Chubukov ${ }^{1,2}$ \\ 1 Condensed Matter Theory Center, Department of Physics, \\ University of Maryland, College Park, Maryland, 20742 \\ ${ }^{2}$ Department of Physics, University of Wisconsin, 1150 University Ave, Madison, WI 53706
}

\begin{abstract}
We study the normal state and pairing instability in electron-doped cuprates near optimal doping. We show that the fermionic self-energy has a non-Fermi liquid form leading to peculiar frequency dependencies of the conductivity and the Raman response. We solve the pairing problem and demonstrate that $T_{c}$ is determined by the curvature of the Fermi surface, and the pairing gap $\Delta(\mathbf{k}, \omega)$ is strongly non-monotonic along the Fermi surface. The normal state frequency dependencies, the value of $T_{c} \sim 10 \mathrm{~K}$ and the $\mathbf{k}$-dependence of the gap agree with the experiment.

PACS: 74.25.-q, 74.20.Mn
\end{abstract}

Physics of high-temperature superconductors continues to attract considerable attention. The past research primarily focused on hole-doped cuprates, where a complex phase diagram was found with antiferromagnetic and superconducting phases separated by a pseudogap region, and where the normal (non-pseudogap) phase displays a prominent non-Fermi-liquid behavior.

Recently there has been a surge of interest in electrondoped cuprates, $\mathrm{Nd}_{2-x} \mathrm{Ce}_{x} \mathrm{CuO}_{4}$ and $\operatorname{Pr}_{2-x} \mathrm{Ce}_{x} \mathrm{CuO}_{4}$. Their phase diagram [1] has a wider region of antiferromagnetism (AFM); superconducting $T_{c}(x)$ forms a dome above the antiferromagnetic quantum-critical point (QCP) [2], much like in heavy fermion materials [3]; and reported pseudogap behavior [4] tracks the Neel temperature, and so is likely just a property of a quasi-2D antiferromagnet [5, 6]. Optical data show [7] the sharp chargetransfer gap near $2 \mathrm{eV}$ at small $x$ melting away near the optimal doping $x \sim 0.15$, where the superconductivity appears. We infer from these data and the experimental phase diagram that near optimal doping the Mott physics is not crucial, and the system behavior can be grasped by considering only low-energy electrons that interact via collective bosons [8]. Electron-boson models have been previously applied to hole-doped cuprates [9, 10], where Mott physics is more pronounced. They must therefore be tested on electron-doped materials. In particular, they should explain a much smaller $T_{c}$ in electron-doped cuprates despite almost the same strength of the Hubbard interaction as in hole-dopes materials [1].

Confinement of the superconductivity to the vicinity of an antiferromagnetic QCP implies that the most likely candidates for the pairing bosons are the spin fluctuations with momenta near the antiferromagnetic vector $\mathbf{Q}=(\pi, \pi)$. RPA studies suggest [12] that AFM appears close to the doping when $2 k_{F}$ coincides with $Q$, i.e. when the Fermi surface (FS) touches the boundary of the AFM Brillouin zone at $\mathbf{k}_{F}=(\pi / 2, \pi / 2)$ (see Fig. 1). Near this point the free-fermion static susceptibility $\chi(\mathbf{Q})$ rapidly increases, leading to the Stoner instability.

Below we set $Q=2 k_{F}$ and consider a spin-fluctuation model for the $2 k_{F}$ instability that involves fermions near $\mathbf{k}_{F}$ and their collective spin fluctuations near Q. Such a model qualitatively differs from the spin-fermion model for hole-doped cuprates [10], where relevant fermionic states (hot spots) are located far from the zone diagonals. In that case, the spin-fluctuation exchange led to the $d_{x^{2}-y^{2}}$-wave pairing [9] with the gap maxima at the hot spots [10]. When the dominant interaction is between the nodal fermions, it is a priori unclear to what degree the $d_{x^{2}-y^{2}}$-wave pairing survives.

In this paper we address this and other issues for $2 k_{F}$ instability. We confirm earlier result 13] that the interaction with low-energy spin fluctuations destroys Fermi liquid behavior for diagonal fermions. We show that this causes a peculiar behavior of the optical conductivity $\sigma(\omega) \sim \omega^{-0.64}$ over a wide frequency range, and a nearly frequency-independent Raman response in the $B_{2 g}$ channel. Both of the results agree with the experiment [14,15]. We then use the normal state results as an input and consider for the first time the spin-mediated pairing near $2 k_{F}$ instability. We demonstrate that $T_{c}$ is finite at the QCP and the pairing is dominated by non Fermi-liquid fermions. We show that the pairing problem is very peculiar as now the curvature of the fermionic dispersion plays a major role. We found $T_{c} \sim 10 \mathrm{~K}$ for the same parameters as for hole-doped materials. We also found non-monotonic variation of the $d$-wave gap $\Delta(k, \omega)$ along the FS (see Fig [4). Non-monotonic variation of the gap agrees with the numerical studies [16] and with the earlier BCS-type study [17], but our solution is very different from the BCS one. The nonmonotonic $d$-wave gap has been detected experimentally at smaller $x$ and has been traced to the location of the hot spots [18, 19]. Our results show that near a magnetic QCP, the positions of the gap maxima deviate from the hot spots, and remain fixed even when the hot spots merge and then disappear.

We now provide the details of the calculations. A spin fermion model describes low-energy fermions $c_{\mathbf{k}}$ interacting with their collective bosonic fluctuations in the spin channel $\mathbf{S}_{\mathbf{q}}: \mathcal{H}_{\text {int }}=g \sum_{\mathbf{k}, \mathbf{q}} c_{\mathbf{k}+\mathbf{q}, \alpha} \boldsymbol{\sigma}_{\alpha \beta} c_{\mathbf{k} \beta} \mathbf{S}_{\mathbf{q}}$ (for internal consistency of the model it is required that $g \lesssim E_{F}[10]$ ). The spin subsystem is described by the static spin susceptibility which, as before, we assume to have the Ornstein- 


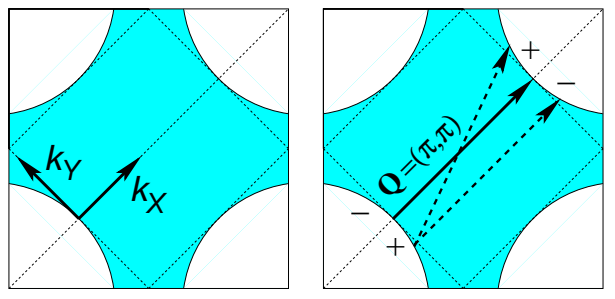

Figure 1: Left: Fermi surface at the antiferromagnetic QCP with $2 k_{F}=(\pi, \pi)$. Diamond-shaped dashed lines bound the magnetic Brillouin zone. The diagonal points of the FS (nodal points of the $d_{x^{2}-y^{2}}$-wave gap) now become "hot". Right: Graphic explanation of the attraction in the $d_{x^{2}-y^{2}}$ channel: parts of the Fermi surface on the same side of the zone diagonals are on average closer to the $\mathbf{Q}$ separation than the parts on the opposite sides, leading to attraction in the $d_{x^{2}-y^{2}}$ channel (plus and minus are the signs of the $d_{x^{2}-y^{2}}$ gap).

Zernike form $\chi_{0}(\mathbf{q})=\chi_{0} /\left[\xi^{-2}+(\mathbf{q}-\mathbf{Q})^{2}\right]$. Interacting fermions are located near $\mathbf{k}_{F}$ and $\mathbf{k}_{F}+\mathbf{Q}$, and have almost antiparallel velocities:

$$
\epsilon_{\mathbf{k}} \approx v_{F} \Delta k_{x}+\beta^{2} \Delta k_{y}^{2}, \quad \epsilon_{\mathbf{k}+\mathbf{Q}} \approx-v_{F} \Delta k_{x}+\beta^{2} \Delta k_{y}^{2},
$$

where $\Delta \mathbf{k}=\mathbf{k}-\mathbf{k}_{F}$. Factor $\beta$ accounts for the curvature of the Fermi line. The strategy is to solve selfconsistently for the normal state bosonic $\Pi(\mathbf{q}, \Omega)$ and fermionic $\Sigma(\mathbf{k}, \omega)$ self-energies, and then use the full normal state propagators as input for the pairing problem. We define the fermionic propagator as $G(\mathbf{k}, \omega)^{-1}=$ $i(\omega+\Sigma(\mathbf{k}, \omega))-\epsilon_{\mathbf{k}}$ and bosonic as $\chi(\mathbf{q}, \omega)^{-1}=\chi_{0}^{-1}(\mathbf{q})+$ $2[\Pi(\mathbf{q}, \omega)+\Pi(2 \mathbf{Q}-\mathbf{q}, \omega)]$.

Normal state Fermionic and bosonic self-energies in the normal state have been obtained by Altshuler et al. [13] within Eliashberg theory, and we just quote their result: at the $2 k_{F}$ antiferromagnetic QCP and $k=k_{F}$ along the diagonal,

$$
\Pi(\mathbf{Q}, \Omega)=\frac{\bar{g}}{2 \pi v_{F} \beta} \sqrt{|\Omega|}, \quad \Sigma\left(\mathbf{k}_{F}, \omega\right)=\omega_{0}^{1 / 4}|\omega|^{3 / 4},
$$

where $\bar{g}=g^{2} \chi_{0}$, and $\omega_{0}=\left(\bar{g} \beta / \pi N v_{F}\right)^{2}$. Both self-energies differ from the case when the hot spots are far from zone diagonals. E.g., $\Pi(\mathbf{q}, \Omega)$ scales as $|\Omega|^{1 / 2}$ instead of the conventional Landau damping form $\Pi(\mathbf{q}, \Omega) \propto|\Omega|$. This is a consequence of antiparallel velocities at $\mathbf{k}_{F}$ and $\mathbf{k}_{F}+\mathbf{Q}$ (observe that the damping rate $\Pi(q, \Omega) \propto 1 / \beta$ does not diverge only because the curvature $\beta \neq 0$ ). Eq. 2 is strictly speaking valid for $\omega>\omega_{0}$. For $\omega<\omega_{0}$ expressions for the self-energies are more involved [13], and one has to sum up series of logarithms to obtain the proper exponents. Altshuler et al. found $\Sigma(\omega) \sim \omega_{0}^{\alpha} \omega^{1-\alpha}$, where $\alpha \sim 0.14$. We confirmed their result. This low-frequency behavior, however, is less important for practical purposes, since we found that $\omega_{0} \sim 10 \mathrm{meV}$ is quite small [20]. Relevant physics, including the pairing, then mostly comes from frequencies $\omega>\omega_{0}$, where Eqs. (2) are valid.
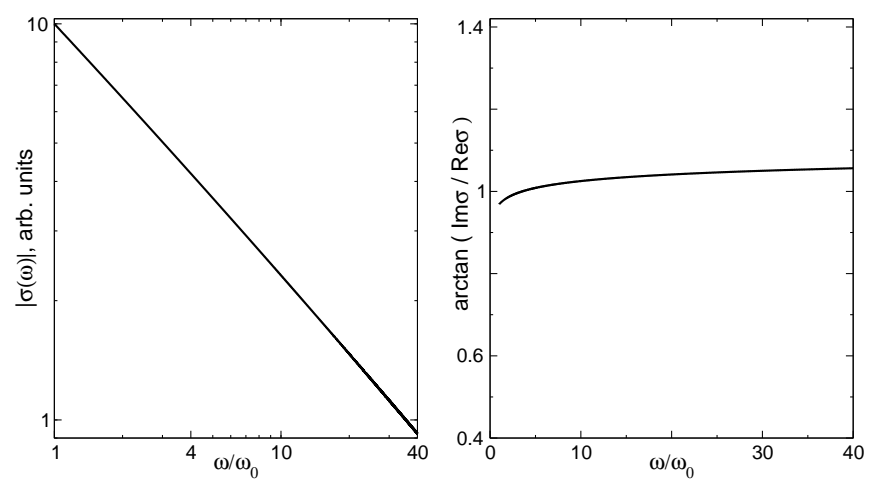

Figure 2: Normal state conductivity as a function of frequency shows a scaling behavior for $\omega_{0}<\omega<40 \omega_{0}$. Left: $|\sigma(\omega)|$, the behavior is indistinguishable from the $\sigma(\omega) \sim \omega^{-0.64}$ dependence. Right: $\arctan \Im \sigma(\omega) / \Re \sigma(\omega)$, an almost constant value means that both $\Im \sigma(\omega)$ and $\Re \sigma(\omega)$ scale as $\omega^{-0.64}$.

For the FS momenta away from the diagonal $\left(k_{y} \neq 0\right.$, see Fig. (1), the non-Fermi liquid form of the self-energy (22) survives up to $k_{y, \max } \sim \omega^{1 / 4}$, and crosses over to the Fermi liquid form at larger $k_{y}$. At the same time, for $\mathbf{k}$ transverse to the FS the self-energy is regular already at $k_{y}=0: \Sigma\left(k_{x}, 0\right) \propto k_{x}$.

Optical conductivity We used the result for the selfenergy to compute complex conductivity $\sigma(\omega)$ from the Kubo formula appropriate when the self-energy mainly depends on frequency. We verified that the dominant contribution to $\sigma(\omega)$ comes from nodal regions where $\Sigma(\omega)$ has a non-Fermi-liquid form [21]. At very small frequencies, $\omega<\omega_{0}, \Sigma(\omega)>\omega$, and both $\Im \sigma$ and $\Re \sigma$ have the same power-law behavior: $\sigma(\omega) \propto \omega^{-1+2 \alpha} \sim \omega^{-0.7}$. At larger frequencies, $\Sigma(\omega)<\omega$, and one should not generally expect $\Re \sigma$ and $\Im \sigma$ to scale with each other. Surprisingly, the scaling behavior extends, with almost the same power, $\sigma(\omega) \propto \omega^{-0.64}$ up to $\sim 40 \omega_{0}$ (see Fig. 21). Such power-law behavior is not a sign of a true scaling, but rather a consequence of the fact that $\Sigma(\omega) / \omega \propto\left(\omega_{0} / \omega\right)^{1 / 4}$ is a slow decaying function. The $\omega^{-\gamma}$ behavior of conductivity with $\gamma \approx 0.68$ has been observed in $\operatorname{Pr}_{1.85} \mathrm{Ce}_{0.15} \mathrm{CuO}_{4}$ below $400 \mathrm{meV}$ 15. Both the exponent and the experimental frequency range are quite consistent with our results. Note that a very similar behavior of the conductivity at intermediate energies has been earlier observed in hole-doped cuprates [22].

Raman response We also considered the Raman scattering in the $B_{2 g}$ channel. Raman intensity is proportional to the imaginary part of the polarization bubble weighted with the geometry dependent Raman form factors $\gamma_{k}$. In the $B_{2 g}$ geometry, $\gamma(\mathbf{k}) \propto \sin k_{x} \sin k_{y}$, such that the signal comes primarily from around the diagonal direction. Unlike the current vertex for conductivity, the Raman vertex is renormalized by the interaction even when $\Sigma(\omega)$ depends only on frequency. Because $\gamma(\mathbf{k})$ is a scalar and doesn't change sign under $\mathbf{k} \rightarrow \mathbf{k}+\mathbf{Q}$, we can 


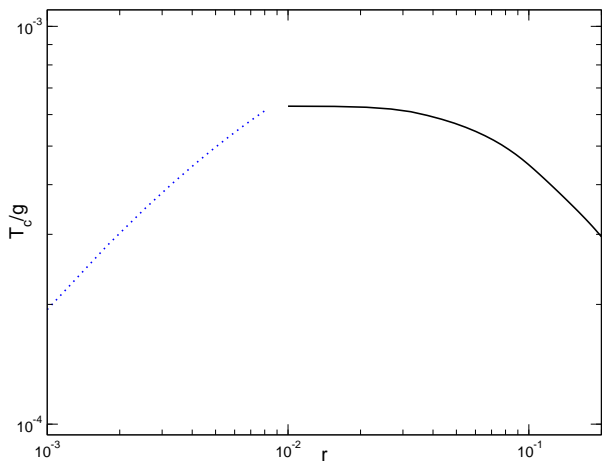

Figure 3: Pairing instability temperature $T_{c}$ in units of the coupling constant $\bar{g}$ vs. the curvature parameter $r=$ $\bar{g} \beta^{2} / \pi v_{F}^{2}$. Solid line gives the result of the numeric solution, which requires increasingly long computational time as $r \rightarrow 0$. Dashed line is the analytic small- $r$ form $T_{c}=$ $0.95 \bar{g} r /\left(1+5 r^{2 / 5}\right)^{4}$.

approximate it by a constant $\gamma\left(\mathbf{k}_{F}\right)$. The renormalization of the Raman vertex then coincides with that of the density vertex, and is related to the self-energy by the Ward identity: the full $\gamma_{\text {full }}(\mathbf{k}, \omega)=\gamma(\mathbf{k})\left(1+\partial_{\omega} \Sigma(\omega)\right)$. Evaluating the Raman bubble and substituting $\gamma_{\text {full }}$ into it, we then obtain

$$
R_{B_{2 g}}(\omega) \sim k_{y, \max }(\omega)\left(1+\partial_{\omega} \Sigma(\omega)\right)^{2} \frac{\omega}{\omega+\Sigma(\omega)}
$$

Substituting the forms of the self-energy and $k_{y, \max }(\omega) \sim$ $\omega^{\alpha}$ for $\omega<\omega_{0}, k_{y, \max }(\omega) \sim \omega^{1 / 4}$ for $\omega>\omega_{0}$, we find that at $T=0, R_{B_{2 g}}(\omega)$ is flat: it is a constant at $\omega<\omega_{0}$, and very slowly crosses over to the $\left(\omega / \omega_{0}\right)^{-1 / 4}$ behavior at $\omega>\omega_{0}$. The flat form of the Raman intensity at frequencies $\omega>\omega_{0}$ is consistent with the experimental data [14]. The experimental behavior at $\omega \leq \omega_{0}$ is dominated by temperature effects which we do not consider.

Pairing instability We now apply normal state results to study the pairing instability near a magnetic QCP. As we said in the introduction, it is a priori unclear whether $d$-wave pairing survives when the hot spots merge, as the strongest pairing interaction involves quasiparticles for which the $d_{x^{2}-y^{2}}$ superconducting gap vanishes. However, separation between near-nodal fermions with opposite signs of the $d_{x^{2}-y^{2}}$ gap is on average closer to $\mathbf{Q}$ than that between fermions with the same sign of the gap (see Fig. 1 b). So the spin-fluctuation exchange still leads to attraction in the $d$-wave channel. Furthermore, as the gap equation relates $\Delta(\mathbf{k})$ near $\mathbf{k}_{F}$ and $\mathbf{k}_{F}+\mathbf{Q}$, where both gaps are linear in the deviation from the nodal direction, the gap equation becomes an equation on the slope of $\Delta(\mathbf{k})$ and does not contain any extra smallness.

The linearized gap equation at QCP is obtained using Eliashberg technique for collective-mode mediated pairing [10]. We verified that the dominant contribution to the pairing comes from $\omega>\omega_{0}$, where $\Sigma(\omega)$ can be approximated by the $\omega^{3 / 4}$ form. To single out the role of the

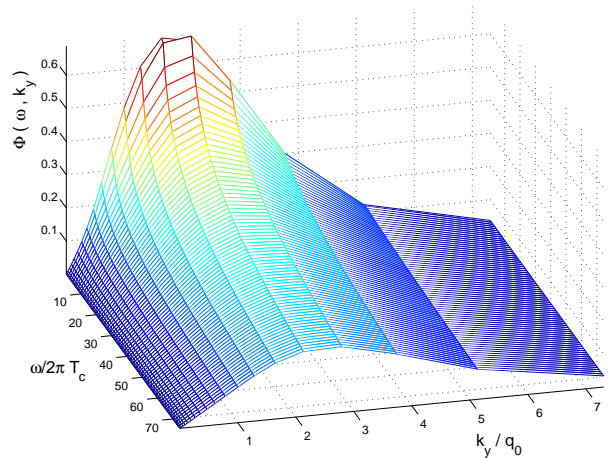

Figure 4: The pairing vertex $\Phi\left(k_{y}, \omega_{n}\right)$ for $r=0.05$ vs $\omega_{n} /\left(2 \pi T_{c}\right)$ and $k_{y} / q_{0}$ ( $q_{0}$ is defined in the text). Observe that $\Phi\left(k_{y}, \omega_{n}\right)$ is non-monotonic in $k_{y}$.

curvature, we rewrite $\omega_{0}$ as $\omega_{0}=\omega_{1} r$, where $\omega_{1}=\bar{g} /(4 \pi)$ accounts solely for the interaction, and the dimensionless $r=\bar{g} \beta^{2} / \pi v_{F}^{2} \sim \bar{g} / E_{F}$ parameterizes the effect of the curvature. Introducing dimensionless variables $k / q_{0}, \omega / \omega_{1}$, where $q_{0}=\bar{g} /\left(\pi v_{F} r^{1 / 4}\right) \sim k_{F}\left(\bar{g} / E_{F}\right)^{3 / 4}$, and assuming that the gap has $d_{x^{2}-y^{2}}$ symmetry, i.e., $\Delta\left(\mathbf{k}_{F}\right)=-\Delta\left(\mathbf{k}_{F}+\mathbf{Q}\right)$, we obtain an integral equation for the pairing vertex $\Phi\left(k_{y}, \omega_{n}\right)$ near a single hot spot:

$$
\begin{aligned}
\Phi\left(k_{y}, \omega_{n}\right) & =\frac{3}{4} T \sum_{\omega_{m}} \frac{r^{1 / 4}}{\left|\omega_{m}\right|+r^{1 / 4}\left|\omega_{m}\right|^{3 / 4}} \\
& \times \int \frac{\Phi\left(k_{y}^{\prime}, \omega_{m}\right) d k_{y}^{\prime}}{\left(k_{y}-k_{y}^{\prime}\right)^{2}+r^{3 / 2}\left(k_{y}^{2}+k_{y}^{\prime 2}\right)^{2}+\sqrt{\left|\omega_{n-m}\right|}} .
\end{aligned}
$$

The momentum dependence in the kernel in (4) comes from $\left(\mathbf{k}-\mathbf{k}^{\prime}\right)^{2}$ after the substitution $k_{x}=\beta^{2} k_{y}^{2} / v_{F}$ and $k_{x}^{\prime}=-\beta^{2} k_{y}^{\prime 2} / v_{F}$. The pairing vertex is related to $\Delta$ as

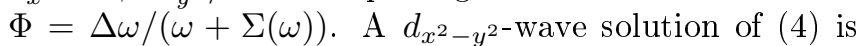
antisymmetric in $k_{y}$ and symmetric in $\omega$ [23].

At vanishing $r$, one can neglect the curvature of the fermionic dispersion in the gap equation (the $r^{3 / 2}$ term in the denominator in (4)). Without this term, the kernel in (4) is non-Fredholmian, and its antisymmetric-in- $k$ solution is a monotonic $\Phi\left(k_{y}, \omega_{n}\right)=k_{y} \Phi\left(\omega_{n}\right)$. Substituting this form into (4), we obtain for $\Phi\left(\omega_{n}\right)$ :

$$
\Phi\left(\omega_{n}\right)=\frac{3}{4} \pi T \sum_{m \neq n} \frac{\omega_{0}^{1 / 4} \Phi\left(\omega_{m}\right)}{\left|\omega_{n-m}\right|^{1 / 4}\left(\left|\omega_{m}\right|+\omega_{0}^{1 / 4}\left|\omega_{m}\right|^{3 / 4}\right)} .
$$

Expectedly (since we neglected the curvature), this equation is the same as in the Eliashberg theory for the pairing, mediated by a bosonic susceptibility $\chi(\omega) \propto \omega^{-1 / 4}$ [24]. Numerical solution of (5) gives $T_{c 1} \approx 6 \omega_{0}=6 \omega_{1} r$, i.e. $T_{c} \sim r$. We emphasize that the $r \rightarrow 0$ limit is not weak coupling (self-energy cannot be neglected) despite the factor $r^{1 / 4}$ in the numerator of (4).

When the curvature of the fermionic dispersion is included, Eq. (4) becomes Fredholmian, and a numerical solution can be obtained using the Nystrom discretization method [25]. We found that the effect of 
the curvature on $T_{c}$ is very strong: above $r>0.001$, the actual $T_{c}$ rapidly becomes much smaller than $T_{c 1}$. For $r \sim 0.1, T_{c} \sim 0.01 T_{c 1}$. We plotted $T_{c}(r)$ in Fig. 3. We see that over a wide range $0.01<r<0.1$, $T_{c} \sim 0.006 \omega_{1} \approx 0.0005 \bar{g}$ forms a plateau and weakly depends on $r$. Using the same $\bar{g} \sim 1.6 \mathrm{eV}$ as for hole-doped cuprates [26], we obtained in this range $T_{c} \sim 10 \mathrm{~K}$. Using further the previous estimate of $\omega_{0}=\omega_{1} r \sim 10 \mathrm{meV}$ we obtain $r \sim 0.08$, which is within the region where $T_{c}$ is almost a constant. Out $T_{c} \sim 10 K$ at a magnetic QCP is in agreement with the experiment [1, 5, 14]. Note that the experimental $T_{c}$ increases in the antiferromagnetic phase up to $20 \mathrm{~K}$, and only then drops at smaller dopings.

The huge discrepancy between $T_{c}$ and $T_{c 1}$ can also be understood analytically, by expanding $T_{c}$ in $r$ beyond the $O(r)$ term. We found that the expansion is rather non-trivial and holds in fractional powers of $r^{2 / 5}: T_{c} \approx$ $T_{c 1} /\left(1+a r^{2 / 5}\right)^{4}$. The prefactor $a>0$ cannot be obtained analytically, but the fit to the numerical data gives $a \sim 5$. As $\left(1+5 r^{2 / 5}\right)^{4}$ rapidly increases with $r$ (it is $\sim 80$ for $r=0.1$ ), this explains why $T_{c}$ is much smaller than $T_{c 1}$.

We also found that at finite $r$ the gap is a nonmonotonic function of $k_{y}$ : it is linear at small $k_{y}$, passes through a maximum at a $k_{0}$ and then falls off (see Fig. 4). Position of the maximum depends on $r$ and appears uncorrelated with the positions of hot spots, which at the QCP are on the zone diagonals. At small $r$ we found analytically that $k_{0}(r) \sim k_{F} r^{3 / 5} \sim k_{F}\left(\bar{g} / E_{F}\right)^{3 / 5}$. As $r$ is small, $k_{0}<k_{F}$ and the gap is confined to the vicinity of the zone diagonals.

At deviations from the QCP towards larger dopings, into a paramagnetic phase, $T_{c}$ decreases and eventually disappears. The value of $k_{0}$, however, does not track the decrease of $T_{c}$, i.e., the $d$-wave gap extends over a finite momentum range along the FS even in the overdoped materials. At deviations into the antiferromagnetic phase, the FS evolves into hole and electron pockets, and the locations of $k_{0}$ gradually approach the hot spots.

Our results for $T_{c}$ and the gap survive even when the magnetic correlation length $\xi$ remains finite at the doping where $2 k_{F}=Q$, i.e., when antiferromagnetic QCP is shifted to lower dopings, when the hot spots are away from zone diagonals. We found that the modifications of our results are small as long as $T_{c}>J \xi^{-2}$ where $J$ is the exchange interaction.

Summary We considered the normal state properties and pairing near a $2 k_{F}$ antiferromagnetic QCP and applied the results to electron-doped cuprates at optimal doping. We found that the breakdown of the Fermi-liquid description at QCP leads to peculiar frequency dependences of the conductivity and the $B_{2 g}$ Raman response. We found that the superconducting $T_{c}$ remains finite at the QCP and is of order $10 \mathrm{~K}$ for the same spin-fermion coupling as for hole-doped cuprates. The pairing gap at QCP has $d_{x^{2}-y^{2}}$ symmetry, but is highly anisotropic and confined to momenta near the zone diagonals.
We acknowledge useful discussions with G. Blumberg, R. Greene, D. Drew, C. Homes, A. Millis, M. Norman, M. M. Qazilbash, and V. Yakovenko. We thank G. Blumberg, A. Millis and M. M. Qazilbash for the comments on the manuscript. The research is supported by Condensed Matter Theory Center at UMD (P.K, A.C) and by NSF DMR 0240238 (A.C.).

[1] Y Tokura, H. Takagi, S. Uchida, Nature 337, 345 (1989).

[2] Y. Dagan et al., Phys. Rev. Lett. 92, 167001 (2004).

[3] P. Coleman and C. Pepin, Acta Physica Polonica B 34, 691 (2003).

[4] A. Koitzsch et al., Phys. Rev. B 67, 184522 (2003).

[5] A. Zimmers et al., Europhys. Lett. 70, 225 (2005).

[6] L. Alff et al., Nature 422, 698 (2003).

[7] Y. Onose et al., Phys. Rev. B 69, 024504 (2004).

[8] R.S. Markiewicz, in Intrinsic Multiscale Structure and Dynamics in Complex Electronic Oxides, ed. by A.R. Bishop, et al., World Scientific (2003).

[9] D.J. Scalapino, Phys. Rep. 250, 329 (1995); P. Monthoux and D. Pines, Phys. Rev. B 47, 6069 (1993).

[10] A. Abanov et al., Adv. Phys. 52, 119 (2003).

[11] A.J. Millis et al., cond-mat/0411172 (unpublished).

[12] F. Onufrieva and P. Pfeuty, Phys. Rev. Lett. 92, 247003 (2004).

[13] B.L. Altshuler, L.B. Ioffe, A.J. Millis, Phys. Rev. B 52, 5563 (1995). The self-energy at finite temperature has been obtained by O. Tchernyshyov and A.V. Chubukov (unpublished). The case of a nested Fermi surface has been analyzed by A. Virosztek and J. Ruvalds, Phys. Rev. B 42, 4064 (1990).

[14] M.M. Qazilbash et al., cond-mat/0510098 to be published in PRB.

[15] C. Homes, unpublished

[16] K. Yoshimura and Hirashima, J. Phys. Soc. Jpn. 74, 712 (2005) and references therein.

[17] V.A. Khodel et al., Phys. Rev. B 69, 144501 (2004).

[18] G. Blumberg et al., Phys. Rev. Lett. 88, 107002 (2002); ibid. 90, 149702 (2003).

[19] H. Matsui et al., Phys. Rev. Lett. 95, 017003 (2005).

[20] We used the same parameters as for hole-doped materials [26] $g=0.7 \mathrm{eV}, \chi_{0} \sim 3 \mathrm{eV}\left(\bar{g}=g^{2} \chi_{0} \sim 1.6 \mathrm{eV}\right)$, and $t-t^{\prime}$ model that fits the ARPES data for Bi2212 to get $v_{F}$ and $\beta$. Note that an analogous scale in hole-doped materials is $\sim 250 \mathrm{meV}[10]$.

[21] This is valid at $T=0$ and finite frequencies, when shortcircuiting of the conductivity (R. Hlubina, T.M. Rice, Phys. Rev. B 51, 9253 (1995)) is not important.

[22] D. van der Marel et al., Nature 425, 271 (2003).

[23] A symmetric in $k_{y}$ solution of (4) that would correspond to a $p$-wave gap stipulates a different overall sign before the kernel; and an odd-frequency $d$-wave solution has lower $T_{c}$ than the even-frequency one.

[24] Ar. Abanov et al. (unpublished).

[25] W.H. Press et al, Numerical Recipes: The Art of Scientific Computing, New York, 2002.

[26] Ar. Abanov et al., Phys. Rev. Lett, 89, 177002 (2002).

[27] A.V. Chubukov, M.R. Norman, Phys. Rev. B 70, 174505 (2004). 